\title{
INTERCALATED MATERIALS
}

\section{Papers sought for 1983 Thermal Society syposium}

The 1983 Symposium on Thermal Behavior of Intercalated Materials will be held Sept. $25-29,1983$, as part of the Twelfth Annual Conference of the North American Thermal Analysis Society.

Symposium chairman--and MRS member--D.D.L. Chung, Department of Metallurgical Engineering and Materials Science, Carnegie-Mellon University, said the meeting will provide an interdisciplinary forum for scientists and engineers interested in the thermal behavior of the intercalation compounds of graphite, transition metal dichalcogenides and polymers. Applications include electrical conductors, battery electrodes, chemical reagents, catalysts and thermal insulators, among others. Aspects of thermal behavior include phase transitions, thermal stability, specific heat, thermal expansion and thermal conductivity at high and low temperatures. The relationships of the thermal properties with electronic, chemical, mechanical and other properties will likewise be explored.

\section{Papers solicited}

Chung said papers on fundamental and applied topics are solicited. Twso hundred word abstracts should be sent to her by Feb. 28. CarnegieMellon University is located in Pittsburgh, Pennsylvania. The Zip Code is 15213. Chung's telephone number is (412) 578-2710.

She noted that manuscripts for publication in the conference proceedings are due May 23. Publication in the proceedings does not preclude subsequent publication elsewhere.

Intercalated materials are of particular interest to many MRS members, Chung noted. At the MRS annual meeting in November, a symposoium on Intercalated Graphite will be chaired by M.S. Dresselhaus,
M.J. Moran and J.E. Fischer.

\section{The Thermal conference}

The joint Twelfth North American Thermal Analysis Society Conference and Calorimetry Conference will be held Sept. 25 - 29, 1983, at the Williamsburg Hilton, Williamsburg, Virginia.

Other symposia on the program include Liquid Crystals and Liquid Crystalline Polymers; Physical Aging Processes in Molecular and Atomic Glasses; Applications of Calorimetry and Thermal Analysis to Catalyst Studies; Thermochemical Studies on Fossil Fuels; Multiphase Polymers and Composites; and Combination Techniques in Thermal Analysis.

For more information about the conference, write to the program chairman, A.R. McGhie, University of Pennsylvania, L.R.S.M., 3231 Walnut Street, Philadelphia, Pennsylvania 19104, or call him at (215) 898-6461. 